\title{
Total replacement of the ascending aorta without circulatory arrest
}

\author{
Marcio Scorsin, MD \\ Nawwar Al-Attar, MD ${ }^{a}$ \\ Marco Scarci, MD \\ Michele Di Mauro, MD ${ }^{\mathrm{b}}$ \\ Richard Raffaul, MD $^{\mathrm{a}}$ \\ Arrigo Lessana, $\mathrm{MD}^{\mathrm{a}}$ \\ Antonio Maria Calafiore, $M D^{\mathrm{b}}$
}

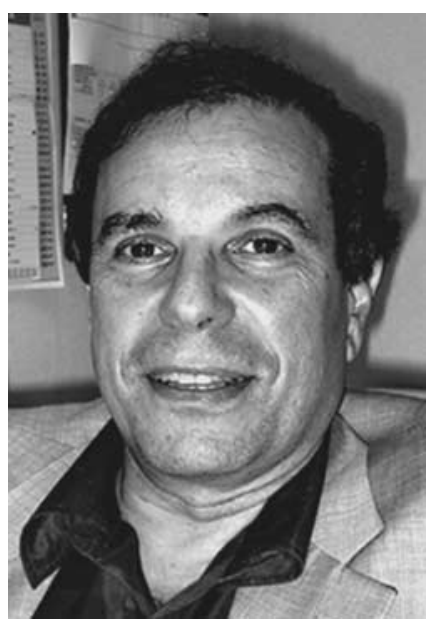

Dr Calafiore
From the Departments of Cardiac Surgery ${ }^{a}$ and Cardiology, ${ }^{\mathrm{b}}$ G. D'Annunzio University, Chieti, Italy.

Received for publication April 17, 2002; revisions requested June 12, 2002; revisions received July 8, 2002; accepted for publication July 12, 2002.

Address for reprints: Antonio Maria Calafiore, MD, G. D'Annunzio University, Division of Cardiac Surgery, S. Camillo de' Lellis Hospital, via C. Forlanini, 50, 66100 Chieti Italy (E-mail: calafiore@unich.it).

J Thorac Cardiovasc Surg 2003;125:126-8

Copyright $(\odot 2003$ by The American Association for Thoracic Surgery

$0022-5223 / 2003 \$ 30.00+0$

doi: $10.1067 / \mathrm{mtc} .2003 .81$
Objective: If the aortic arch is clamped between the brachiocephalic trunk and the left common carotid artery and the brachiocephalic trunk is also clamped, the total ascending aorta can be replaced without circulatory arrest. Candidates for this technique need to have the following characteristics: preoperative demonstration of Willis polygon patency by means of transcranial Doppler ultrasonography, preoperative computed tomographic scan that shows no calcification in the arch or in the brachiocephalic trunk, and preoperative or intraoperative evidence of separate origins of the brachiocephalic trunk and the left common carotid artery.

Methods: In three different institutions (with different time frames in each) from December 2000 to December 2001, the possibility of replacing the total ascending aorta without circulatory arrest was evaluated in 14 cases. In 10 of them it was possible (feasibility of 71.4\%). During the procedure continuous electroencephalographic monitoring was performed.

Results: No patient died in the early or midterm follow-up. None of the patients had any cerebral complications. The electroencephalographic activity was normal during the period of brachiocephalic trunk occlusion. In 5 patients a postoperative spiral computed tomographic scan showed good surgical results.

Conclusions: The technique described here allows replacement of the total ascending aorta without circulatory arrest in selected cases. Furthermore, the evidence of lack of right cerebral hypoperfusion in all the procedures we performed allows future exploration of the possibility of avoiding cerebral monitoring.

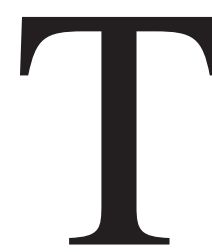

otal replacement of the ascending aorta is generally obtained with a short period of circulatory arrest at various temperatures with and without antegrade or retrograde cerebral perfusion. We describe here a technique (as theorized by M.S.) that in selected patients allows replacement of the entire ascending aorta in normothermia without any circulatory arrest.

\section{Material and Methods \\ Background}

In about $95 \%$ of cases the Willis polygon is widely anatomically and functionally open. ${ }^{1}$ This ensures the perfusion of the right side of the brain through the left carotid artery and the left vertebral artery. With a simple examination (transcranial Doppler), patency of Willis polygon can be detected. In these patients, the brachiocephalic trunk (BCT) can be occluded during a surgical procedure without reduction of right cerebral flow. Maintenance of sufficient flow can be monitored by continuous electroencephalographic activity recording. The ability to occlude the BCT allows crossclamping of the aortic arch between the BCT and the origin of 


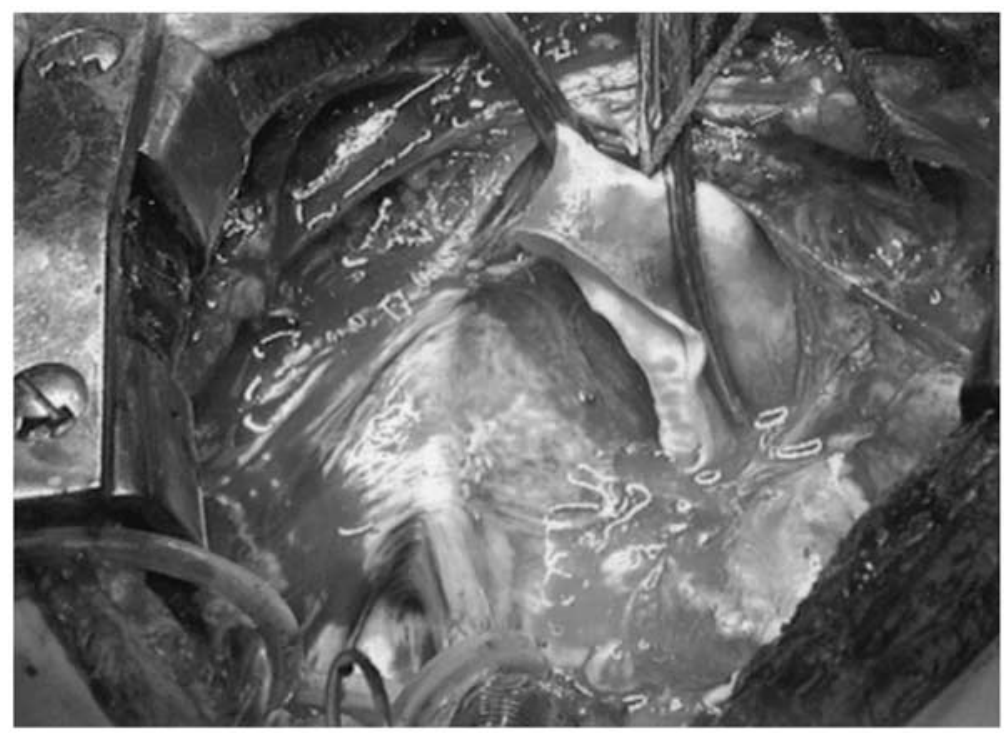

Figure 1. Intraoperative view (from below). BCT is occluded and ascending aorta is resected.

the left common carotid artery (LCCA), making possible the replacement of the whole ascending aorta without any circulatory arrest.

\section{Patient Selection}

Candidates for this technique are patients who need total ascending aorta replacement and have the following characteristics: preoperative transcranial Doppler ultrasonography that demonstrates Willis polygon patency, preoperative computed tomographic scan that shows no calcification in the arch or in the BCT, preoperative or intraoperative evidence of separate origins of the BCT and the LCCA, and preoperative or perioperative evidence of extensive atheroma in the aortic arch wall.

\section{Surgical Technique}

The patient is monitored as usual, with added leads to record the electroencephalographic activity. The femoral artery is cannulated as well as both venae cavae or the right atrium directly, according the presence or absence of an additional cardiac procedure. The aortic arch is dissected, and the BCT is encircled with a tape. During the first part of the procedure the ascending aorta is crossclamped as usual, and the BCT is occluded for 20 minutes to evaluate the effectiveness of the single LCCA-left vertebral artery perfusion. When necessary, the aortic arch is pulled proximally to better expose its middle portion and is crossclamped between the $\mathrm{BCT}$ and the LCCA. The BCT is again occluded and the ascending aorta resected (Figure 1). A tubular graft of appropriate size is sewn with a polypropylene suture (Figure 2).

\section{Clinical Experience}

From December 2000 to December 2001, a total of 10 patients in three different institutions (with different time frames in each location) underwent total ascending aorta replacement with the technique described here. In 4 other investigated cases the technique could not be applied because of abnormal origin of LCCA from the BCT in 3 cases and arch calcifications in 1 case. Table 1 shows preoperative data. In the same period, 4 patients had aortic arch surgery and 24 had regular distal ascending aorta clamping for partial ascending aorta replacement.

\section{Results}

Perioperative and postoperative data are shown in Table 2. Applicability of the technique was $71.4 \%(n=10 / 14)$. In addition to total ascending aorta replacement, 6 patients underwent aortic valve replacement, 1 underwent aortic root replacement, and 2 underwent coronary artery bypass grafting. No patients died in the early or midterm follow-up periods. None of them had any cerebral complications. The electroencephalographic activity was normal during the period of BCT occlusion, demonstrating that right brain perfusion through the left carotid and vertebral arteries is safe if the Willis polygon is properly working. Spiral computed tomographic scans performed in 5 patients showed good surgical results.

\section{Discussion}

Total ascending aorta replacement is generally accomplished with a short period of circulatory arrest. Even if this strategy is safe, cardiopulmonary bypass time is increased and the patient is exposed to some potential complications, such as organ malfunction, excessive bleeding, and cerebral edema or focal lesions. ${ }^{2}$ Furthermore, patients with borderline dilated aortas associated with aortic valve pathologic conditions sometimes to avoid circulatory arrest do not undergo replacement of the total ascending aorta, because the potential complications are not justified by clear literature data. The technique described here, which has not 


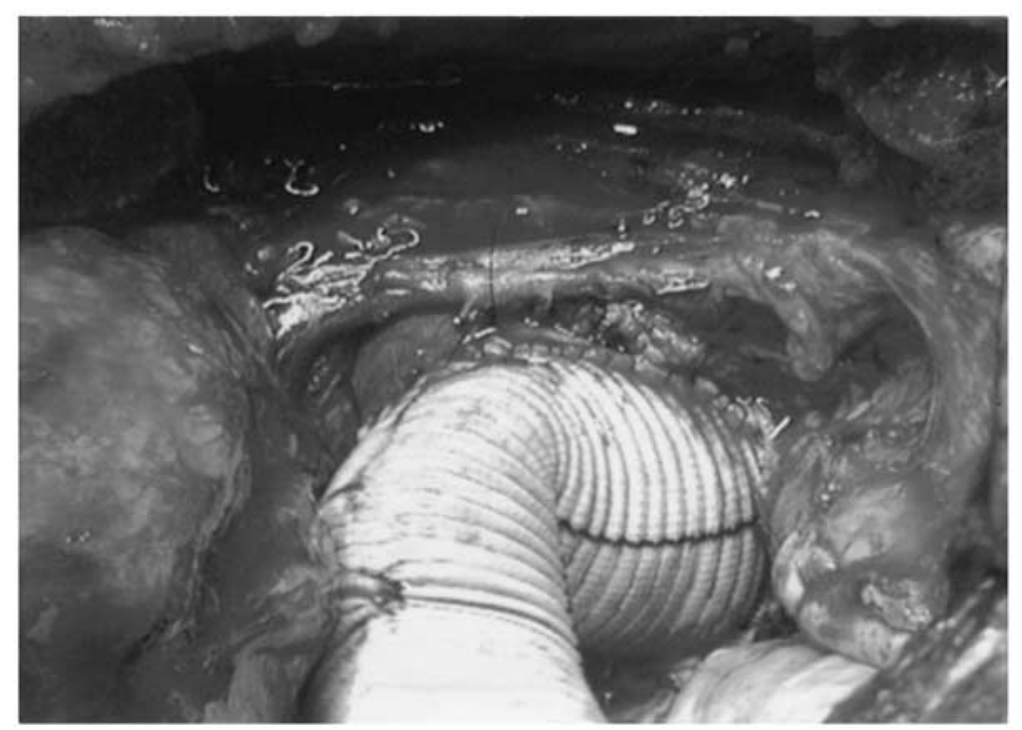

Figure 2. Intraoperative view (from below). Tubular graft of appropriate size is sewn.

TABLE 1. Preoperative data $(n=10)$

\section{Age (y)}

Female sex (No.)

Hypertension (No.)

Aortic valve stenosis (No.)

Aortic valve regurgitation (No.)

Coronary disease (No.)

Ejection fraction (\%)

previously been reported to the best of our knowledge, allows in selected cases replacement of the total ascending aorta during a conventional normothermic or mildly hypothermic cardiopulmonary bypass provided that the Willis polygon works properly (if not, selective perfusion of innominate artery, directly or through the right subclavian artery, can be considered). The use of electroencephalographic monitoring may be cumbersome, but the procedure is done on an elective basis and thus its timing can be easily adapted to the necessities of the neurologist. Furthermore, the evidence of lack of right cerebral hypoperfusion in all the operations that we performed allows us to explore the possibility of avoiding cerebral monitoring in the future.
TABLE 2. Perioperative and postoperative data $(n=10)$

Aortic crossclamp time (min)

Cardiopulmonary bypass time (min)

Reoperations for bleeding (No.)

Awaking time (h)

Ventilation time (h)

Time to extubation (h)

Deaths (No.)

Cerebrovascular accidents (No.)

Acute renal failure (No.)

Bleeding (mL/12 h)

Transfused patients (No.)

Intensive care unit stay (h)

Hospital stay (d)

\section{References}

1. Hoksbergen AW, Legemate DA, Ubbink DT, Jacobs MJ. Collateral variations in circle of Willis in atherosclerotic population assessed by means of transcranial color-coded duplex ultrasonography. Stroke. 2000;31:1656-60.

2. Svensson LJ, Crawford ES, Hess KR, Coselli JS, Raskin S, Shenaq SA, et al. Deep hypothermia with circulatory arrest. J Thorac Cardiovasc Surg. 1993;106:19-31. 